\title{
INTERPOLATION AND UNIQUENESS RESULTS FOR ENTIRE FUNCTIONS
}

\author{
BY \\ JAMES D. CHILD(1)
}

\begin{abstract}
Let $K[\Omega]$ denote the collection of entire functions of exponential type whose Borel transforms are analytic on $\Omega^{c}$ (the complement of the simply connected domain taken relative to the sphere). Let $f$ be in $K[\Omega]$ and set $L_{n}(f)=(2 \pi i)^{-1} \int_{\Gamma} g_{n}(\lambda) F(\lambda) d \lambda(n=0,1, \ldots)$ where $F$ is the Borel transform of $f, \Gamma \subset \Omega$ is a simple closed contour chosen so that $F$ is analytic outside and on $\Gamma$ and each $g_{n}$ is in $H(\Omega)$ (the collection of functions analytic on $\Omega$ ). In what follows read 'the sequence of linear functionals $\left\{L_{n}(f)\right\}$ ' wherever the sequence of functions ' $\left\{g_{n}\right\}$ ' appears. Let $T$ denote a continuous linear operator from $H(\Omega)$ to $H(\Lambda)$ where $\Lambda$ is also a simply connected domain. The topologies on $H(\Omega)$ and $H(\Lambda)$ are those of uniform convergence on compact subsets of $\Omega$ (resp. $\Lambda$ ).

The purpose of this paper is to consider uniqueness preserving operators, i.e., operators $T$ which have the property that $K[\Lambda]$ is a uniqueness class for $\left\{T\left(g_{n}\right)\right\}$ whenever $K[\Omega]$ is a uniqueness class for $\left\{g_{n}\right\}$, and to examine interpolation preserving operators, i.e., operators $T$ which have the property that $K[\Lambda]$ interpolates the sequence of complex numbers $\left\{b_{n}\right\}$ relative to $\left\{T\left(g_{n}\right)\right\}$ whenever $K[\Omega]$ interpolates $\left\{b_{n}\right\}$ relatives to $\left\{g_{n}\right\}$. Once some classes of uniqueness preserving operators and some classes of interpolation preserving operators have been found, we proceed to obtain new uniqueness and interpolation results from our knowledge of these operators and from previously known uniqueness and interpolation results.

Operators which multiply by analytic functions and some differential operators are considered. Composition operators are studied and the results are used to extend the interpolation results for sequences of functions of the form $\left\{[W(\zeta)]^{n}\right\}$ where $W$ is analytic and univalent on a simply connected domain.
\end{abstract}

1. Introduction. Let $\Omega$ denote a simply connected domain in the complex plane and $K[\Omega]$ the collection of entire functions of exponential type whose

Received by the editors January 23, 1973 and, in revised form, April 4, 1974.

AMS (MOS) subject classifications (1970). Primary 30A66.

Key words and phrases. Entire functions of exponential type, dual space, dual operator, uniqueness class, interpolation results.

(1)Most of these results are contained in the author's $1972 \mathrm{Ph}$. D. dissertation at the University of Cincinnati, written under the supervision of Professor Richard F. DeMar. 
Borel transforms are analytic on $\Omega^{c}$. For each $f$ in $K[\Omega]$ let $B(f)$ denote its Borel transform. Note that $K[\Omega]$ is often thought of as the collection of entire functions of exponential type whose conjugate indicator diagrams are contained in $\Omega$. The class of functions analytic on $\Omega$ will be denoted by $H(\Omega)$. We assume throughout the article that $H(\Omega)$ has the topology of uniform convergence on compact subsets placed on it. It is well known [16, pp. 35, 38] that $K[\Omega]$ may be identified with the dual space of $H(\Omega)$. In fact $H(\Omega)$ is also the dual space of $K[\Omega]$. The bilinear form pairing them is given by

$$
\langle\alpha, g\rangle=(2 \pi i)^{-1} \int_{\Gamma} \alpha(\lambda) G(\lambda) d \lambda,
$$

where $\alpha$ is in $H(\Omega), g$ is in $K[\Omega]$ with $G=B(g)$, and $\Gamma \subset \Omega$ is a simple closed contour chosen so that $G$ is analytic outside and on $\Gamma$.

The uniqueness and interpolation problems with which we shall deal involve sequences $\left\{L_{n}\right\}$ of continuous linear functionals defined on $K[\Omega]$. From (1.1) it is clear that there exists a sequence $\left\{g_{n}\right\}$ of functions from $H(\Omega)$ so that

$$
L_{n}(f)=\left\langle g_{n}, f\right\rangle \quad(n=0,1, \ldots) .
$$

Throughout this article we shall identify each sequence of continuous linear functionals on $K[\Omega]$ with the sequence of functions from $H(\Omega)$ which generate them as in (1.2). With this identification we say that $K[\Omega]$ is a uniqueness class for $\left\{g_{n}\right\}$ if $f$ in $K[\Omega]$ and $\left\langle g_{n}, f\right\rangle=0(n=0,1, \ldots)$ imply that $f=0$. We note that such sequences $\left\{g_{n}\right\}$ are also said to be total on $K(\Omega)$. A sequence of complex numbers $\left\{b_{n}\right\}$ is said to be interpolated by $K[\Omega]$ relative to $\left\{g_{n}\right\}$ if there exists $f$ in $K[\Omega]$ with $\left\langle g_{n}, f\right\rangle=b_{n}(n=0,1, \ldots)$.

Much of the previous work done in the area of uniqueness and interpolation involves sequences of the form $\left\{[W(\zeta)]^{n}\right\}$ where $W$ is a function analytic on $\Omega$. An easy application of our theory gives a new proof of known necessary and sufficient conditions for $K[\Omega]$ to be a uniqueness class for $\left\{[W(\zeta)]^{n}\right\}$. Our theory also yields an improved theorem giving necessary and sufficient conditions for $K[\Omega]$ to interpolate $\left\{b_{n}\right\}$ relative to $\left\{[W(\zeta)]^{n}\right\}$. The theory described in what follows examines characterizations of certain types of operators which 'preserve' the notion of uniqueness as well as others that 'preserve' the notion of interpolation. These characterizations will be used to examine certain simple, but useful, operators which will in turn be used to obtain new uniqueness and interpolation results from old ones. We fix the ideas above with the following definitions. Let $\Lambda$ denote a simply connected domain and let $T$ denote a continuous linear operator from $H(\Omega)$ to $H(\Lambda)$. Then $T$ is said to be uniqueness preserving if $K[\Lambda]$ is a uniqueness class for $\left\{T\left(g_{n}\right)\right\}$ whenever $K[\Omega]$ is a uniqueness class for $\left\{g_{n}\right\}$. The 
operator $T$ is said to be interpolation preserving if $K[\Lambda]$ interpolates $\left\{b_{n}\right\}$ relative to $\left\{T\left(g_{n}\right)\right\}$ whenever $K[\Omega]$ interpolates $\left\{b_{n}\right\}$ relative to $\left\{g_{n}\right\}$.

2. Some general results. Let $T$ denote a continuous linear operator from $H(\Omega)$ to $H(\Lambda)$. For each $f$ in $K[\Omega]$ define $T^{\prime}(f)$ by

$$
\left\langle\alpha, T^{\prime}(f)\right\rangle=\langle T(\alpha), f\rangle \text { for all } \alpha \text { in } H(\Omega) .
$$

The operator $T^{\prime}$ from $K[\Lambda]$ to $K[\Omega]$ defined above is called the dual operator of $T$. This dual operator plays an important part in the results which follow.

The following easy theorems give useful characterizations of the operators under consideration. The proofs which are omitted below can be found in [7]

THEOREM 2.2. Let $T$ be a continuous linear operator from $H(\Omega)$ to $H(\Lambda)$. The following conditions are equivalent.

(a) $T$ is uniqueness preserving,

(b) $T^{\prime}$ is one-to-one,

(c) $T(H(\Omega))$ is dense in $H(\Lambda)$, and

(d) there exists a sequence $\left\{g_{n}\right\}$ of functions from $H(\Omega)$ so that $K[\Lambda]$ is a uniqueness class for $\left\{T\left(g_{n}\right)\right\}$.

THEOREM 2.3. Let $T$ be a continuous linear operator from $H(\Omega)$ to $H(\Lambda)$. Then the following conditions are equivalent.

(a) $T$ is interpolation preserving,

(b) $T^{\prime}$ maps onto $K[\Omega]$, and

(c) there exists a sequence $\left\{g_{n}\right\}$ such that $K[\Omega]$ is a uniqueness class for $\left\{g_{n}\right\}$ and $K[\Lambda]$ interpolates $\left\{b_{n}\right\}$ relative to $\left\{T\left(g_{n}\right)\right\}$ whenever $K[\Lambda]$ interpolates $\left\{b_{n}\right\}$ relative to $\left\{g_{n}\right\}$.

Proof ((c) implies (a)). Assume $T$ is not interpolation preserving, then by the equivalence of (a) and (b) $T^{\prime}$ does not map onto $K[\Omega]$. Let $g$ be in $K[\Omega]$ so that $g$ is not in the image of $K[\Lambda]$ under $T^{\prime}$. Set $b_{n}=\left\langle g_{n}, g\right\rangle(n=0,1, \ldots)$. By hypothesis there exists $f$ in $K[\Lambda]$ so that $\left\langle T\left(g_{n}\right), f\right\rangle=b_{n}(n=0,1, \ldots)$. But, then $\left\langle g_{n}, g\right\rangle=\left\langle g_{n}, T^{\prime}(f)\right\rangle(n=0,1, \ldots)$ and $g \neq T^{\prime}(f)$ which contradicts the hypothesis that $K[\Omega]$ is a uniqueness class for $\left\{g_{n}\right\}$. Hence, $T$ must be interpolation preserving.

THEOREM 2.4. If $T$ is a continuous linear operator from $H(\Omega)$ to $H(\Lambda)$, then the following conditions are equivalent.

(a) $T$ is both uniqueness preserving and interpolation preserving,

(b) $T$ is a linear homeomorphism onto $H(\Lambda)$,

(c) $T$ and $T^{\prime}$ are both one-to-one, and

(d) $T$ maps onto $H(\Lambda)$ and $T^{\prime}$ maps onto $K[\Omega]$. 
3. A further characterization of uniqueness preserving operators. Since the spaces dealt with here are nice, the continuous linear functions discussed above have a useful integral representation. It is known [16] that there is a one-to-one correspondence between the continuous linear operators, denoted by $T$, from $H(\Omega)$ to $H(\Lambda)$ and the functions denoted by $M(\lambda, \zeta)$ which are locally holomorphic on $\Omega^{c} \times \Lambda$ and which vanish at $\infty$ for each $\zeta$ in $\Lambda$. This correspondence is described as follows. For each $\alpha$ in $H(\Omega)$ and each $\zeta$ in $\Lambda$

$$
[T(\alpha)](\zeta)=(2 \pi i)^{-1} \int_{\Gamma} \alpha(\lambda) M(\lambda, \zeta) d \lambda,
$$

where $\Gamma \subset \Omega$ is a simple closed contour depending upon the particular $\zeta$ which is chosen so that $M(\lambda, \zeta)$ is analytic outside and on $\Gamma$. We remark that $M(\lambda, \zeta)=$ $1 / \lambda-\zeta$ generates the identity operator from $H(\Omega)$ to $H(\Omega)$. In this case (3.1) is just the Cauchy integral formula. In this section we will characterize the locally holomorphic functions which generate uniqueness preserving operators as in (3.1). More directly we shall characterize a class of functions on $\mathrm{C} \times \Lambda$ which yield an integral representation for $T^{\prime}$. These functions are described below.

Suppose that $M(w, \zeta)$ is locally holomorphic on $\Omega^{c} \times \Lambda$ and vanishes at $\infty$ for each $\zeta$ in $\Lambda$, then for each $\zeta$ in $\Lambda, M(\lambda, \zeta)$ is the Borel transform of some function in $K[\Omega]$. Thus, the Pólya representation of entire functions of exponential type may be used to define $m(w, \zeta)$ as follows. For each $\zeta^{\prime}$ in $\Lambda$

$$
m(w, \zeta)=(2 \pi i)^{-1} \int_{\Gamma} e^{w \lambda} M(\lambda, \zeta) d \lambda
$$

for all $\zeta$ in some disk $D\left(\zeta^{\prime}, r\right) \subset \Lambda$, where $\Gamma \subset \Omega$ is chosen so that $M(\lambda, \zeta)$ is analytic outside and on $\Gamma$ for all $\zeta$ in $D\left(\xi^{\prime}, r\right)$ and so that $M(\lambda, \zeta)$ is analytic on $D\left(\zeta^{\prime}, r\right)$ for each $\lambda$ on $\Gamma$. It follows that $m(w, \zeta)$ is holomorphic on $\mathbf{C} \times \Lambda$ and that $m(w, \zeta)$ is in $K[\Omega]$ for each $\zeta$ in $\Lambda$. It can be shown by direct methods that if $T$ and $M$ are related as in (3.1), $T^{\prime}$ and $m$ are related by

$$
\left[T^{\prime}(f)\right](w)=(2 \pi i)^{-1} \int_{\Gamma_{\zeta}} m(w, \zeta) F(\zeta) d \zeta
$$

for each $f$ in $K[\Lambda]$, where $\Gamma_{\zeta} \subset \Lambda$ is chosen so that $F=B(f)$ is analytic outside and on $\Gamma_{\zeta}$. It is relatively simple to determine $m$ given $M$ using (3.2). For example if $M(\lambda, \zeta)=1 / \lambda-\zeta$ and $\Omega=\Lambda$ then $m(w, \zeta)=e^{\zeta w}$ and $T^{\prime}$ determined by (3.3) is the identity map from $K[\Omega]$ to $K[\Omega]$. The next theorem characterizes the functions $m$ which correspond by (3.1) and (3.2) to uniqueness preserving operators $T$.

THEOREM 3.4. Let $T$ be a continuous linear operator from $H(\Omega)$ to $H(\Lambda)$ and suppose $m(w, \zeta)$ is related to $T$ by expressions (3.1) and (3.2). For each 
$n=0,1, \ldots$ set $g_{n}(\zeta)=\left.\left[\partial^{n} m(w, \zeta) / \partial w^{n}\right]\right|_{w=0}$. Then $T$ is uniqueness preserving if and only if $K[\Lambda]$ is a uniqueness class for $\left\{g_{n}\right\}$.

Proof. Suppose that $T$ is uniqueness preserving and that $f$ is in $K[\Lambda]$ with $\left\langle g_{n}, f\right\rangle=0(n=0,1, \ldots)$. Set $h(w)=(2 \pi i)^{-1} \int_{\Gamma} m(w, \zeta) F(\zeta) d \zeta ;$ then for each $n=0,1, \ldots$

$$
h^{(n)}(0)=\left.(2 \pi i)^{-1} \int_{\Gamma}\left[\partial^{n} m(w, \zeta) / \partial w^{n}\right]\right|_{w=0} F(\zeta) d \zeta=\left\langle g_{n}, f\right\rangle=0,
$$

Hence, $h=0$ so that $T^{\prime}$ one-to-one implies that $f=0$.

Suppose that $K[\Lambda]$ is a uniqueness class for $\left\{g_{n}\right\}$. Let $f$ be in $K[\Lambda]$ with $T^{\prime}(f)=0$. Then for each $n=0,1, \ldots$,

$$
0=\partial^{n}\left[T^{\prime}(f)\right](w) /\left.\partial w^{n}\right|_{w=0}=(2 \pi i)^{-1} \int_{\Gamma} g_{n}(\zeta) F(\zeta) d \zeta, \quad B(f)=F .
$$

By hypothesis $f=0$ and $T^{\prime}$ is one-to-one. Thus, $T$ is uniqueness preserving.

The class of functions $m(w, \zeta)$ holomorphic on $\mathbf{C} \times \Lambda$ with the property that $f=0$ whenever $f$ is in $K[\Lambda], B(f)=F$, and $\int_{\Gamma} m(w, \zeta) F(\zeta) d \zeta=0$ was suggested to be of interest by DeMar in [15].

4. A further characterization of uniqueness preserving and interpolation preserving operators. Let $U$ denote the unit disk with center the origin. We shall link operators which are both uniqueness preserving and interpolation preserving from $H(U)$ to $H(U)$ to the notion of proper basis in $H(U)$. The sequence $\left\{\alpha_{n}\right\}$ of functions in $H(U)$ is said to be a proper basis in $H(U)$ if for each $\alpha$ in $H(U)$ there exists a sequence of complex numbers $\left\{c_{n}\right\}$ șo that $\Sigma_{n=0}^{\infty} c_{n} \alpha_{n}(\zeta)$ converges to $\alpha(\zeta)$ for all $\zeta$ in $U$, if $c_{n}=0(n=0,1, \ldots)$ whenever $\Sigma_{n=0}^{\infty} c_{n} \alpha_{n}(\zeta)$ converges to 0 on $U$, and if $\Sigma_{n=0}^{\infty} c_{n} \alpha_{n}(\zeta)$ converges on $U$ if and only if $\Sigma_{n=0}^{\infty} c_{n} \zeta^{n}$ converges on $U$. Some examples of proper bases may be found in [1]. We now state our characterization.

THEOREM 4.1. Let $T$ be a continuous linear operator from $H(U)$ to $H(U)$ and let $m(w, \zeta)$ be related to $T$ by (3.1) and (3.2). Set $g_{n}(\zeta)=\partial^{n} m(w, \zeta) /\left.\partial w^{n}\right|_{w=0}$ $(n=0,1, \ldots)$. Then $T$ is both uniqueness preserving and interpolation preserving if and only if $\left\{g_{n}\right\}$ is a proper basis in $H(U)$.

Proof ('if'). By [2, Theorem 1] since $\left\{g_{n}\right\}$ is a proper basis in $H(U)$, for each $r_{0}$ with $0<r_{0}<1$

$$
\limsup _{n \rightarrow \infty}\left[\max _{|\zeta|=r_{0}}\left|g_{n}(\zeta)\right|\right]^{1 / n}<1 .
$$

Hence, for each positive $r_{0}<1$ there is a $\rho_{0}<1$ so that $\Sigma_{n=0}^{\infty} g_{n}(\zeta) / \lambda^{n+1}$ con. 
verges for each $\zeta$ with $|\zeta|<r_{0}$ and for each $\lambda$ with $|\lambda|>\rho_{0}$. Thus, for each $\zeta$ in $U, N(\lambda, \zeta)=\Sigma_{n=0}^{\infty} g_{n}(\zeta) / \lambda^{n+1}$ is analytic on $U$ and zero at $\infty$. Set

$$
k(w, \zeta)=(2 \pi i)^{-1} \int_{\Gamma} e^{w \lambda} N(\lambda, \zeta) d \lambda=\sum_{n=0}^{\infty} g_{n}(\zeta) w^{n} / n !
$$

Note that the series in (4.2) converges uniformly on compact subsets of $\mathbf{C}$ for each $\zeta$ in $U$. Then $\partial^{n} k(w, \zeta) /\left.\partial w^{n}\right|_{w=0}=g_{n}(\zeta)(n=0,1, \ldots)$ and for each positive $r_{0}<1$ there is a positive $\rho_{0}<1$ so that $\Sigma_{n=0}^{\infty} g_{n}(\zeta) / \lambda^{n+1}$ converges to $M(\lambda, \zeta)$ corresponding to $m$ for each $\zeta$ with $|\zeta|<r_{0}$ and for each $\lambda$ with $|\lambda|>\rho_{0}$. Fix $r_{0}$ with $0<r_{0}<1$, set $\delta_{n}(\lambda)=\lambda^{n}(n=0,1, \ldots)$ and let $\Gamma$ be a circle with center the origin and radius greater than $\rho_{0}$ corresponding to $r_{0}$ but less than 1. Then for each $n=0,1, \ldots$,

$$
\begin{aligned}
{\left[T\left(\delta_{n}\right)\right](\zeta) } & =(2 \pi i)^{-1} \int_{\Gamma} M(\lambda, \zeta) \delta_{n}(\lambda) d \lambda=(2 \pi i)^{-1} \int_{\Gamma}\left(\sum_{i=0}^{\infty} g_{i}(\zeta) / \lambda^{i+1}\right) \lambda^{n} d \lambda \\
& =\sum_{i=0}^{\infty} g_{i}(\zeta)\left[(2 \pi i)^{-1} \int_{\Gamma} \lambda^{n} / \lambda^{i+1} d \lambda\right]=g_{n}(\zeta) .
\end{aligned}
$$

The uniform convergence on $\Gamma$ of the series was used to interchange the order of integration and summation. Hence, $\Gamma$ generated by $M$ maps $\delta_{n}$ to $g_{n}(n=0,1$, $\ldots$... By [2, Theorem 2] that $\left\{g_{n}\right\}$ is a proper basis in $H(U)$ guarantees the existence of a linear homeomorphism from $H(U)$ onto $H(U)$ which maps $\delta_{n}$ to $g_{n}$ for each $n=0,1, \ldots$ Clearly, $T$ must be this linear homeomorphism and , thus, $T$ is both uniqueness preserving and interpolation preserving.

('only if'). If $T$ is both uniqueness preserving and interpolation preserving, then from Theorem 2.6 $T$ is a linear homeomorphism. By [2, Theorem 2] if $T\left(\delta_{n}\right)=\alpha_{n}(n=0,1, \ldots)$ then $\left\{\alpha_{n}\right\}$ is a proper basis in $H(U)$. But it was shown in the proof of the 'if' part that if $\left\{\alpha_{n}\right\}$ is a proper basis in $H(U), k(w, \zeta)$ $=\Sigma_{n=0}^{\infty} \alpha_{n}(\zeta) w^{n} / n !$, and $T_{k}$ is the continuous linear operator corresponding to $k$, then $\partial^{n} k(w, \zeta) /\left.\partial w^{n}\right|_{w=0}=\alpha_{n}(\zeta)$ and $T_{k}\left(\delta_{n}\right)=\alpha_{n}(n=0,1, \ldots)$. Thus, $T_{k}=$ $T, k(w, \zeta)=m(w, \zeta), \alpha_{n}=g_{n}(n=0,1, \ldots)$, and $\left\{g_{n}\right\}$ is a proper basis in $H(U)$. Thus, the proof is completed.

5. Some applications of the theory. In this section the theory developed above will be applied to consider simple but useful examples of uniqueness preserving operators as well as examples of operators which are both uniqueness preserving and interpolation preserving. Composition operators, operators which multiply by an analytic function, and some differentiation operators will be considered. These operators will then be used to produce uniqueness and interpolation results. We remark that once our machinery has been developed one may quickly 
and easily obtain known results that required much work to obtain in the first place. The machinery also leads directly to new results. The following discussion provides examples of the simplicity and usefulness of our method.

Let $\Lambda$ be a simply connected domain in the complex plane. Set $\Omega=W(\Lambda)$ where $W$ is a function analytic on $\Lambda$ and define $T$ from $H(\Omega)$ to $H(\Lambda)$ by [T( $\alpha)](\zeta)=\alpha(W(\zeta))$ for each $\alpha$ in $H(\Omega)$. If $W$ is univalent on $\Lambda$ then $T$ is a linear homeomorphism so that by Theorem $2.4 T$ is both uniqueness preserving and interpolation preserving. However, if $W$ is not univalent on $\Lambda$ with $W\left(\zeta_{1}\right)=$ $W\left(\zeta_{2}\right)$ for $\zeta_{1} \neq \zeta_{2}$ then every $\beta$ in the range of $T$ has the property $\beta\left(\zeta_{1}\right)=\beta\left(\zeta_{2}\right)$ so that $T$ does not have dense range. By Theorem $2.2 T$ is not uniqueness preserving. The preceding discussion is summarized by the next theorem.

THEOREM 5.1. The operator $T$ corresponding to $W$ analytic on $\Lambda$ is both uniqueness preserving and interpolation preserving if and only if $W$ is univalent on $\Lambda$.

This theorem encompasses two theorems proved by DeMar [11, Theorem 2, p. 8] and [18, Lemma 1]. Many of the sequences of functions analytic on a simply connected domain $\Lambda$ which have been previously studied are of the form $\left\{[W(\zeta)]^{n}\right\}$ where $W$ is analytic and univalent on $\Lambda$. The next result follows from the fact that $K[\mathrm{C}]$ is a uniqueness class for $\left\{\lambda^{n}\right\}$ since $\left\{\lambda^{n}\right\}$ corresponds to the sequence of functionals $\left\{f_{(0)}^{(n)}\right\}$.

Corollary 5.2. Let $W$ be analytic on $\Lambda$. A necessary and sufficient condition for $K[\Lambda]$ to be uniqueness class for $\left\{[W(\zeta)]^{n}\right\}$ is that $W$ is univalent on $\Lambda$.

This result is not new but it has a long history. See e.g. Gel'fond [13], Buck [6], and DeMar [10]. Our proof is simpler. The following corollary is an improvement over other attempts to find such necessary and sufficient conditions by Buck [5] and DeMar [8] and [9] since it is not required that the origin be in $\Lambda$ or that $W(0)=0$ if the origin is in $\Lambda$. Its proof may be found in [7].

COROLlary 5.3. Let $W$ be analytic and univalent on $\Lambda$. Let $\left\{b_{n}\right\}$ be a sequence of complex numbers and define a function $B_{0}$ by $B_{0}(\zeta)=\Sigma_{n=0}^{\infty} b_{n} / \zeta^{n+1}$ and its analytic continuation. Then $K[\Lambda]$ interpolates $\left\{b_{n}\right\}$ relative to $\left\{[W(\zeta)]^{n}\right\}$ if and only if $B_{0}$ is analytic on $[W(\Lambda)]^{c}$.

EXAMPLE 5.4. Previous attempts at determining the necessary and sufficient conditions in Corollary 5.3 did not cover the Carlson functionals $\{f(n)\}$ since they are generated by the sequence $\left\{e^{n \zeta}\right\}$ so that $W(\zeta)=e^{\zeta}$ which does not have the property that $W(0)=0$. Set $\Lambda=\{\zeta=x+i y:-\pi<y<\pi\}$. Then $W(\Lambda)$ is 
the complex plane slit from the origin to $\infty$ along the negative $x$-axis. Hence, $\left\{b_{n}\right\}$ can be interpolated by $K[\Lambda]$ relative to $\{f(n)\}$ if and only if $B_{0}(\zeta)=$ $\Sigma_{n=0}^{\infty} b_{n} / \zeta^{n+1}$ is analytic on $[W(\Lambda)]^{c}$. Note that a similar result holds if $\Lambda$ is taken to be $\{\zeta=x+i y: \pi<y<3 \pi\}$ which does not contain the origin.

It may be of interest to the reader to note that the integral formulas discussed in $\S \S 3$ and 4 applied to the composition operator $T$ corresponding to $W(\zeta)$ and its dual $T^{\prime}$ are given by

$$
\begin{gathered}
{[T(\alpha)](\zeta)=(2 \pi i)^{-1} \int_{\Gamma} \alpha(\zeta) /(\lambda-W(\zeta)) d \zeta,} \\
{\left[T^{\prime}(f)\right](z)=(2 \pi i)^{-1} \int_{\Gamma} e^{z W(\zeta)} F(\zeta) d \zeta, \quad F=B(f) .}
\end{gathered}
$$

From the characterization of uniqueness preserving the interpolation preserving operators in $\S 4$, one may observe that if $W$ is analytic and univalent on the unit disk $U$ mapping $U$ onto $U$ then by Theorems 4.1 and $5.1\left\{[W(\zeta)]^{n}\right\}$ is a proper basis in $H(U)$.

We now turn our attention to operators which multiply by a given analytic function. Let $A$ denote a function which is analytic on the simply connected domain $\Omega$. In the following let $T$ denote the linear operator from $H(\Omega)$ to $H(\Omega)$ defined by $T(\alpha)=A \alpha$ for each $\alpha$ in $H(\Omega)$. The uniqueness preserving and interpolation preserving properties of $T$ follow directly.

THEOREM 5.5. If $A \not \equiv 0$, then the corresponding $T$ is interpolation preserving.

Proof. Suppose there exists $g$ in $K[\Omega]$ with $\left\langle g_{n}, g\right\rangle=b_{n}(n=0,1, \ldots)$. Then $(2 \pi i)^{-1} \int_{\Gamma} g_{n}(\lambda) G(\lambda) d \lambda=b_{n}(n=0,1, \ldots)$ where $G=B(g)$ and $\Gamma \subset \Omega$ is a simple closed contour chosen so that $A$ has no zeros on $\Gamma$ and $G$ is analytic outside and on $\Gamma$. Then $G(\lambda) / A(\lambda)$ is analytic on $\Gamma$, hence, there exist functions $F_{i}$ and $F_{0}$ with $G(\lambda) / A(\lambda)=F_{i}(\lambda)+F_{0}(\lambda)$ for all $\lambda$ in some neighborhood of $\Gamma$, where $F_{i}$ is analytic inside and on $\Gamma$ and $F_{0}$ is analytic outside and on $\Gamma$ with $F_{0}(\infty)=0$. Now

$$
\begin{aligned}
b_{n} & =(2 \pi i)^{-1} \int_{\Gamma} g_{n}(\lambda) G(\lambda) d \lambda=(2 \pi i)^{-1} \int_{\Gamma} g_{n}(\lambda)\left[A(\lambda) F_{i}(\lambda)+A(\lambda) F_{0}(\lambda)\right] d \lambda \\
& =(2 \pi i)^{-1} \int_{\Gamma} g_{n}(\lambda) A(\lambda) F_{0}(\lambda) d \lambda .
\end{aligned}
$$

But, $f_{0}$ with $B\left(f_{0}\right)=F_{0}$ is in $K[\Omega]$ and the result follows.

If $A$ has no zeros on $\Omega$ then $T$ is a linear homeomorphism and is, therefore, uniqueness preserving. If $A$ has zeros on $\Omega$, then $T$ cannot have dense range. Thus, we have the following result. 
THEOREM 5.6. The operator $T$ corresponding to $A$ is uniqueness preserving if and only if $A$ is nonzero on $\Omega$.

The following example demonstrates how the preceding two theorems may be used to obtain new uniqueness and interpolation results.

EXAMPLE 5.7. The Abel functionals $\left\{f^{(n)}(n)\right\}$ are generated by the sequences of functions $\left\{\left(\zeta e^{\xi}\right)^{n}\right\}$. Set $\Omega=\left\{r e^{i \theta}: r<(\pi-|\theta|) / \sin |\theta|\right\}$. Then $\zeta e^{\zeta}$ is univalent on $\Omega$ and by Corollary $5.2 K[\Omega]$ is a uniqueness class for $\left\{\left(\zeta e^{\zeta}\right)^{n}\right\}\left[\left\{f^{(n)}(n)\right\}\right]$. Set $A(\zeta)=e^{\zeta}+e^{-\zeta}$, then $A$ has no zeros on $\Omega$ so Theorem 5.6 may be applied to obtain that $K[\Omega]$ is a uniqueness class for the sequence of functions $\left\{\zeta^{n} e^{(n+1) \zeta}+\zeta^{n} e^{(n-1) \xi}\right\}$. Using the traditional terminology, we obtain that $K[\Omega]$ is a uniqueness class for the sequence of linear functionals $\left\{f^{(n)}(n+1)+f^{(n)}(n-1)\right\}$. Applying Theorem 5.5 and Corollary 5.3 we find that $K[\Omega]$ interpolates $\left\{b_{n}\right\}$ relative to $\left\{f^{(n)}(n+1)+f^{(n)}(n-1)\right\}$ if and only if $B_{0}$ defined $B_{0}(\zeta)=\Sigma_{k=0}^{\infty} b_{k} / \zeta^{k+1}$ is analytic on $\Omega^{c}$.

The dual operator $T^{\prime}$ of $T$ defined by $T(\alpha)=A \alpha$ has the integral representation

$$
\left[T^{\prime}(f)\right](w)=(2 \pi i)^{-1} \int_{\Gamma} A(\zeta) e^{\zeta w} F(\zeta) d \zeta, \quad F=B(f)
$$

If $D^{n}=d^{n} / d w^{n}$ and $A(\zeta)=\Sigma_{n=0}^{\infty} a_{n} \xi^{n}$ then formally $\left[T^{\prime}(f)\right](w)=\Sigma_{n=0}^{\infty} a_{n} D^{n} f(w)$. The equation

$$
g(w)=\sum_{n=0}^{\infty} a_{n} D^{n} f(w)
$$

where $f$ and $g$ are in $K[\Omega]$ has been previously studied; see Boas and Buck [4, pp. 67-70] for a discussion and further references. Theorems 5.5 and 5.6 provide simple answers to the questions concerning existence and uniqueness of solutions of (5.8). After making the assumptions on $\Omega$ required by the type of summability used, one can make the following observations. Since $T$ is interpolation preserving, $T^{\prime}$ maps onto $K[\Omega]$, so for each $g$ in $K[\Omega]$, there exists $f$ in $K[\Omega]$ with $g(w) \sim \sum_{n=0}^{\infty} a_{n} D^{n} f(w)$. Such expansions are unique if and only if $A$ has no zeros on $\Omega$.

We conclude by considering some differentiation operators. First, the operator $D$ defined on $H(\Omega)$ for some fixed $\Omega$ has integral representation

$$
[D(\alpha)](\zeta)=(2 \pi i)^{-1} \int_{\Gamma} \alpha(\lambda) /(\lambda-\zeta)^{2} d \lambda
$$

for each $\alpha$ in $H(\Omega)$. Its dual, $D^{\prime}$, has the representation 


$$
\left[D^{\prime}(f)\right](w)=(2 \pi i)^{-1} \int_{\Gamma_{1}} w e^{\zeta w} F(\zeta) d \zeta=w f(w) \quad(F=B(f)) .
$$

Clearly, $D^{\prime}$ is one-to-one on $K[\Omega]$ so that $D$ is uniqueness preserving. The following example demonstrates the value of knowing that the operator $D$ is uniqueness preserving.

EXAMPLE 5.9. Let $\left\{a_{n}\right\}$ be a sequence of complex numbers such that $a_{n} \rightarrow 0$ and $\sum_{n=0}^{\infty}\left|a_{n}-a_{n+1}\right|<\infty$. Gontcharoff [14] has shown that $K[\mathrm{C}]$ is a uniqueness class for the sequence of functionals $\left\{f^{(n)}\left(a_{n}\right)\right\}$. Since $D$ is uniqueness preserving we can say that $K[\mathrm{C}]$ is also a uniqueness class for $\left\{n f^{(n-1)}\left(a_{n}\right)\right.$ $\left.+a_{n} f^{(n)}\left(a_{n}\right)\right\}$.

An application of our theory shows that if $\Omega$ is a simply connected domain containing the origin, then $T$ defined by $[T(\alpha)](\zeta)=\alpha(\zeta)+\zeta \alpha^{\prime}(\zeta)$ is both uniqueness preserving and interpolation preserving from $H(\Omega)$ to $H(\Omega)$. Example 5.10 results from this observation.

EXAMPLE 5.10. Set $\Omega=\{\zeta=x+i y:|y|<\pi\}$ then from Corollary 5.2 we note that $K[\Omega]$ is a uniqueness class for $\{f(n)\}$. From the preceding remarks we observe that $K[\Omega]$ is also a uniqueness class for $\left\{f(n)+n f^{\prime}(n)\right\}$. Using Example 5.4 and the comments preceding this example, one obtains that $\left\{b_{n}\right\}$ can be interpolated by $K[\Omega]$ relative to $\left\{f(n)+n f^{\prime}(n)\right\}$ if and only if $\Sigma_{k=0}^{\infty} b_{k} / \zeta^{k+1}$ is analytic on $\Omega^{c}$.

\section{REFERENCES}

1. M. G. Arsove, The Pincherle basis problem and a theorem of Boas, Math. Scand. 5 (1957), 271-275. MR 20 \#1787.

2. - Proper bases and linear homeomorphisms in spaces of analytic functions, Math. Ann. 135 (1958), 235-243. MR 20 \#7215.

3. R. P. Boas, Jr., Entire functions, Academic Press, New York, 1954. MR 16, 914.

4. R. P. Boas, Jr. and R. C. Buck, Polynomial expansions of analytic functions, Ergebnisse der Mathematik und ihrer Grenzgebiete, N. F., Heft 19, Springer-Verlag, Berlin, 1958. MR 20 \#984.

5. R. C. Buck, Integral valued entire functions, Duke Math. J. 15 (1948), 879-891. MR 10, 693. $10,693$.

6. - Interpolation series, Trans. Amer. Math. Soc. 64 (1948), 283-298. MR

7. J. D. Child, Uniqueness and interpolation results for entire functions of exponential type, Bull. Amer. Math. Soc. 79 (1973), 734-737. MR 47 \#8866.

8. R. F. DeMar, Existence of interpolating functions of exponential type, Trans. Amer. Math. Soc. 105 (1962), 359-371. MR 25 \#5317.

9. - On a theorem concerning existence of interpolating functions, Trans. Amer. Math. Soc. 114 (1965), 23-29. MR 35 \#1788.

10. - A uniqueness theorem for entire functions, Proc. Amer. Math. Soc. 16 (1965), 69-71. MR 30 \#2145.

11. - A study of interpolation series, Final Technical Report N.S.F. Grant \#9619, 1970 (unpublished). 
12. R. E. Edwards, Functional analysis. Theory and applications, Holt, Rinehart and Winston, New York, 1965. MR 36 \#4308.

13. A. O. Gel'fond, Interpolation et unicité des fonctions entières, Mat. Sb. 4 (1938), $115-147$.

14. W. Gontcharoff, Recherches sur les dérivées successives des fonctions analytiques. Généralisation de la série d'Abel, Ann. École Norm. Sup. 47 (1930), 1-78.

15. J. Korevaar et al. (editor), Entire functions and related parts of analysis, Proc. Sympos. Pure Math., vol 11, Amer. Math. Soc., Providence, R. I., 1968. MR 38 \#1258.

16. G. Köthe, Dualität in der Funktionentheorie, J. Reine Angew. Math. 191 (1953), 30-49. MR 15, 132.

17. - Topological vector spaces. I, Die Grundlehren der math. Wissenschaften, Band 159, Springer-Verlag, New York and Berlin, 1969. MR 40 \#1750.

18. H. Shankar (editor), Mathematical essays dedicated to A. J. MacIntyre, Ohio Univ. Press, Athens, Ohio, 1970. MR 42 \#6194.

DEPARTMENT OF MATHEMATICS, ROLLINS COLLEGE, WINTER PARK, FLORIDA 32789 